\title{
The role of the TB T-spot test in patients with tubercular retinal vasculitis
}

\author{
Elin Lee ${ }^{1,3}$, Srinivasan Sanjay ${ }^{1,4}$, Stephen Teoh ${ }^{1,2}$ \\ 'Department of Ophthalmology and Visual Sciences, Khoo Teck Puat Hospital \\ (KTPH), Singapore; ${ }^{2}$ Department of Ophthalmology, Tan Tock Seng Hospital \\ (TTSH), Singapore; ${ }^{3}$ Ministry of Health Holdings (MOHH), Singapore; ${ }^{4}$ Yong Loo Lin \\ School of Medicine, National University of Singapore (YLLSoM, NUS), Singapore
}

\begin{abstract}
Purpose: To report tuberculosis (TB) T-spot diagnosis and management of two cases of presumptive tubercular retinal vasculitis in Singapore.

Method: Retrospective case reports.

Results: Two patients were diagnosed with presumptive TB retinal vasculitis using clinical features, TB T-spot test and ancillary investigations. They were successfully treated with a combination therapy of oral steroids, anti-tuberculosis medications and pan retinal photocoagulation (PRP). Follow-up demonstrated resolution of symptoms and signs with no complications arising from anti-tuberculosis medications.

Conclusions: TB retinal vasculitis presents diagnostic and therapeutic challenges. Ocular $T B$ diagnosis is often presumptive, as confirmatory evidence of organisms from the eye has low yield. The results of culture and Mantoux test require a long waiting period. TB $T$-spot provides rapid results with high sensitivity and specificity, thus holding promise as a diagnostic tool. Management of TB retinal vasculitis is multi-modal and multi-disciplinary requiring oral steroids and pan-retinal photocoagulation by the ophthalmologists and anti-tuberculosis medications by the Physicians.
\end{abstract}

Key words: Tuberculosis, retinal vasculitis, TB T-spot, Singapore

\section{Introduction}

Tuberculosis (TB) is an infectious disease caused by various strains of mycobacteria, commonly Mycobacterium tuberculosis. It typically affects the lungs, but also causes extrapulmonary manifestations in the eye. In 2011, the World Health Organization (WHO) estimated 8.7 million incident cases of TB. ${ }^{1}$ TB is endemic in Singapore, the incidence rate increased from 39.2 in 2010 to 40.5 per 100,000 in 2011. ${ }^{2}$ Extrapulmonary incidence in Singapore accounts for $1.2 \%$ of all TB cases. ${ }^{1}$ Sharma et al. reported ocular TB incidence ranging from 1.4-5.7\%. ${ }^{3}$ Incidence of ocular TB in Singapore is not known. In human immunodeficiency virus (HIV) patients, the incidence may increase to between 2.8-11.4\%. ${ }^{3}$ TB affects many ocular tissues, including retinal vasculitis. ${ }^{4}$ The haematogenous route of spread is the most common. ${ }^{4}$ Retinal vasculitis has been postulated to arise from delayed type hypersensitivity reaction to sensitized tuberculous protein in the vessel walls. ${ }^{5}$

The diagnosis of tubercular vasculitis is often presumptive as there may be no

Correspondence: Dr. Srinivasan Sanjay, Department of Ophthalmology and Visual Sciences, Khoo Teck Puat Hospital, Alexandra Health, 90 Yishun Central, Singapore 768828. E-mail: sanjay_s@alexandrahealth.sg 
confirmatory evidence of tuberculosis in these patients. ${ }^{5}$ To our knowledge and according to Gupta et al., ${ }^{5}$ there are no published studies yet on Interferon-gamma Release Assays (IGRA) (e.g., TB T-spot) and ocular TB. We present a pilot study on two cases of presumptive unilateral TB retinal vasculitis discussing the management and role of TB-T spot in the diagnosis in Singapore.

\section{Materials and Methods}

Two patients with presumptive TB retinal vasculitis were diagnosed and identified by a specialist in an ophthalmology clinic in Singapore. The diagnosis was made according to Gupta et al's ${ }^{5}$ presumptive diagnostic criteria as shown below. Additionally, the TB T-spot test was positive in both cases.

\section{Presumptive ocular TB diagnostic criteria ${ }^{5}$}

Any one or more clinical signs listed in Section I in combination with any positive tests under section II or a positive therapeutic trial section in section III in combination with section IV.

\section{Section I - Clinical presentation in intraocular TB}

\begin{tabular}{|l|l|}
\hline 1. Anterior uveitis & $\begin{array}{l}\text { Granulomatous, nongranulomatous, iris nodules, } \\
\text { ciliary body tuberculoma }\end{array}$ \\
\hline 2. Intermediate uveitis & $\begin{array}{l}\text { Granulomatous, nongranulomatous with } \\
\text { organizing exudates in the pars lana/ peripheral } \\
\text { uvea }\end{array}$ \\
\hline 3. Posterior and panuveitis & $\begin{array}{l}\text { Choroidal tubercule, choroidal tuberculoma, } \\
\text { subretinal abscess, serpiginous-like choroiditis }\end{array}$ \\
\hline 4. Retinitis and retinal vasculitis & $\begin{array}{l}\text { 5. Neuroretinitis and optic } \\
\text { neuropathy }\end{array}$ \\
\hline $\begin{array}{l}\text { 6. Endophthalmitis and } \\
\text { panophthalmitis }\end{array}$ & \\
\hline
\end{tabular}

\section{Section II - Systemic investigations}

1. Positive Mantoux reaction

2. Evidence of healed or active tubercular lesion on radiography of the chest

3. Evidence of confirmed active extrapulmonary TB (either by microscopic examination or by culture of the affected tissue for M. Tuberculosis)

Section III - Therapeutic test

A positive response to four drug anti-tuberculosis treatment (ATT) (Isoniazid, Rifampicin, Ethambutol and Pyrazinamide) over a period of 4 to 6 weeks. 


\section{Section IV - Exclusion of other Uveitis Entities}

In the geographic regions where TB is low in incidence, other causes of uveitis must be excluded by various laboratory investigations including serology for syphilis, toxoplasmosis and others.

\section{Results}

\section{Case 1}

A 36-year-old Chinese man with no past medical history presented with two months of floaters in the left eye associated with low-grade fever and with cough of two months duration, four months prior. He was asymptomatic in the right eye. There was no travel history or prior contact with TB.

Visual acuity (VA) was $6 / 6$ unaided in both eyes. The anterior segments were normal. However, fundus examination of the left eye revealed sheathing of vessels with flame-shaped haemorrhages noted in the superior and temporal quadrants (Fig. 1a). The right fundus was normal. Fundus fluorescein angiography (FFA) (Fig. 2a) showed capillary non-perfusion with perivascular leakage in the areas of haemorrhages. The only positive investigation was a reactive TB T-spot ${ }^{\circledR}(O x f o r d$ Immunotec/ United Kingdom) test. Chest X-ray (CXR) was normal. Additional investigations for infective and autoimmune causes were normal (Table 1). Mantoux test was not performed.

A provisional presumptive diagnosis of TB-associated supero-temporal retinal vasculitis was made. He was commenced on oral Rifampicin $10 \mathrm{mg} / \mathrm{kg}$ OD, Isoniazid $300 \mathrm{mg}$ OD, Ethambutol $15 \mathrm{mg} / \mathrm{kg}$ OD, Pyrazinamide $25 \mathrm{mg} / \mathrm{kg}$ OD for a duration of six months. Oral prednisolone $1 \mathrm{mg} / \mathrm{kg}$ was commenced 48 hours later. Sectoral retinal photocoagulation was applied to the ischaemic areas. The haemorrhages resolved after 11 months with no neovascularisation or complications (Fig. 1b). A presumptive diagnosis of TB-associated retinal vasculitis was subsequently confirmed upon resolution of fundal changes with the four-drug ATT.

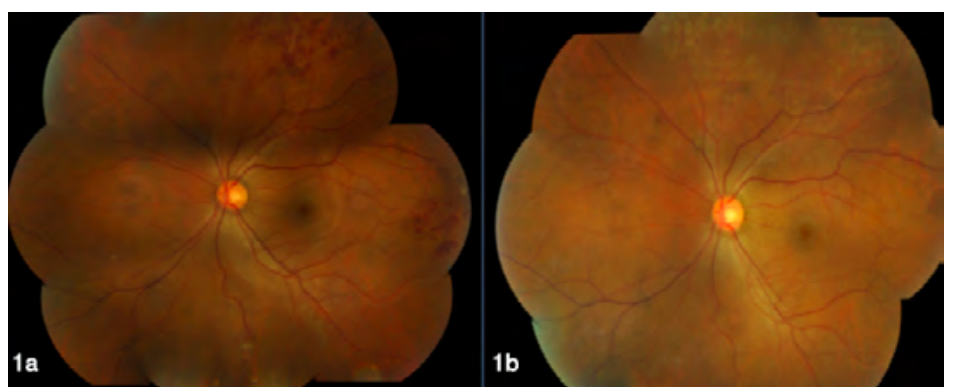

Fig. 1. Mosaic fundal photographs OU (Case 1). a. Superotemoral flame-shaped haemorrhages and sheathing and sclerosis of vessels. b. Resolved flame-shaped haemorrhages with photocoagulation scars, ten months later. 


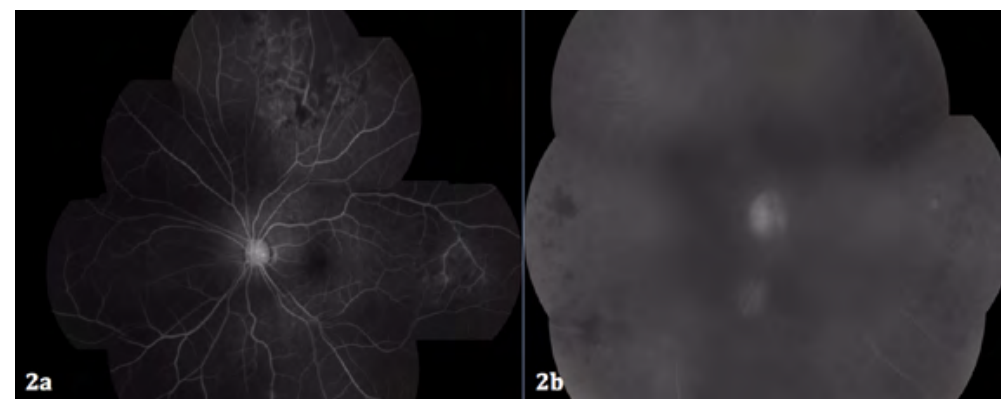

Fig. 2a. FFA OS showing leakage and blocked hypofluorescence and capillary non-perfusion in the superotemporal fundal region (Case 1).

Fig. 2b. FFA OD showing focal vasculitis with no capillary non-perfusion (Case 2).

Case 2

A 58-year-old Chinese man, who was a hepatitis-B carrier on Adefovir, presented with the first episode of right eye floaters of one-week duration. This was associated with on-off joint pains and swelling for one year. He had no rashes, mouth ulcers, loss of appetite or weight, cough or night sweats.

Visual acuity was $6 / 6$ in both eyes. The anterior segments were normal. Dilated right fundus showed preretinal haemorrhage superiorly, cotton-wool spots inferiorly with diffuse clusters of dot-blot haemorrhages and small patches of retinal infiltrates, perivascular sheathing and vitreous clumps along the superior and inferior retinal vessel arcades but not vitritis (Fig. 3a). The left fundus was normal. FFA confirmed focal areas of vasculitis but no areas of capillary non-perfusion (Fig. 2b).

Investigations showed a reactive TB T-spot test and elevated inflammatory markers were raised (ESR $36 \mathrm{~mm} / \mathrm{hr}$, CRP $8.5 \mathrm{mg} / \mathrm{L}$ ). His CXR showed three stable granulomas that were present in previous CXRs. Other investigations for infective and autoimmune causes were normal (Table 1). Mantoux test was not performed.

The presumptive diagnosis of right TB-associated vasculitis was made. A baseline AST and ALT were normal (Table 1). Patient was commenced on oral Rifampicin 10 $\mathrm{mg} / \mathrm{kg}$, Isoniazid $5 \mathrm{mg} / \mathrm{kg}$ and Pyrazinamide $25 \mathrm{mg} / \mathrm{kg}$ for duration of six months. Oral prednisolone $0.5 \mathrm{mg} / \mathrm{kg}$ was commenced 48 hours later. One month later, right eye panretinal photocoagulation (PRP) was done. Symptoms and fundus changes resolved after eight months (Fig. 3b) and patient no longer complained of floaters.

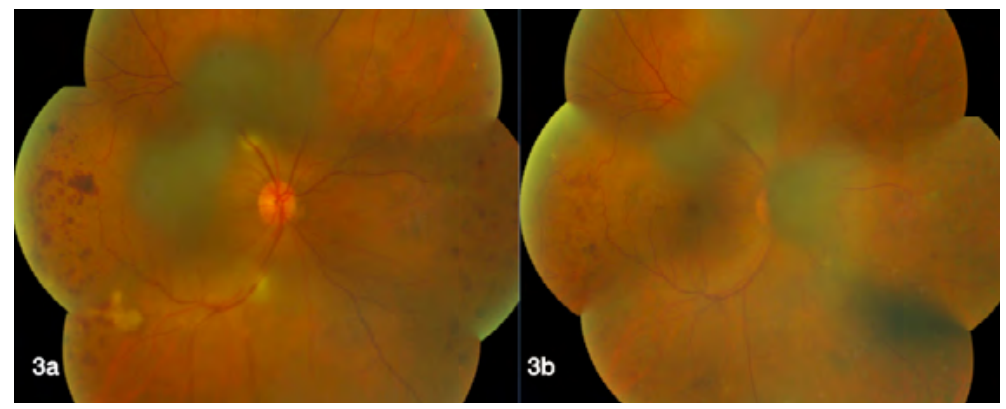

Fig. 3. Mosaic fundal photographs OD (Case 2). a. Dot blot haemorrhages, cotton wool spots and perivascular sheathing. b. Resolved fundal changes with PRP scars eight months later. 
Table 1. Laboratory investigative results of two patients with presumptive tuberculous retinal vasculitis.

\begin{tabular}{|c|c|c|c|}
\hline Laboratory test & Reference range & Case 1 & Case 2 \\
\hline TB T-spot & Nil & Reactive & Reactive \\
\hline Haemoglobin $(\mathrm{g} / \mathrm{dL})$ & $13.0-17.0$ & 14.6 & 10.52 \\
\hline White blood cells $\left(10^{9} / \mathrm{L}\right)$ & $4.00-11.00$ & 5.33 & 14.8 \\
\hline Platelets $\left(10^{9} / \mathrm{L}\right)$ & $130-400$ & 221 & 303 \\
\hline $\begin{array}{l}\text { Erythrocyte sedimentation rate (ESR) (mm/ } \\
\mathrm{hr})\end{array}$ & $1-10$ & 3 & 36 \\
\hline C-reactive protein (CRP) (mg/L) & $1.0-5.0$ & $<1.0$ & 8.5 \\
\hline Rheumatoid factor (RF) (IU/mL) & $<14$ & $<10$ & $<10$ \\
\hline Anti-myeloperoxidase (U/mL) & $0-20$ & $<1$ & $<1$ \\
\hline Anti-PR3 (U/mL) & $0-20$ & $<1$ & 2 \\
\hline Anti-nuclear antibody (ANA) (\%) & $<100$ & 17 & 60 \\
\hline $\begin{array}{l}\text { Anti-double-stranded deoxyriboneuclotide } \\
\text { acid (anti-ds DNA) (IU/mL) }\end{array}$ & $1.0-25.0$ & 0.9 & 6.5 \\
\hline $\begin{array}{l}\text { Rapid plasma regain/venereal disease } \\
\text { research laboratory (RPR/VDRL) }\end{array}$ & N.A. & \multicolumn{2}{|c|}{ Non-reactive } \\
\hline Anti- hepatitis C virus (anti-HCV) & N.A. & \multicolumn{2}{|c|}{ Non-reactive } \\
\hline Hepatitis B surface antigen (HBsAg) & N.A. & $\begin{array}{l}\text { Non- } \\
\text { reactive }\end{array}$ & Reactive \\
\hline $\begin{array}{l}\text { Human immunodeficiency syndrome } \\
\text { antigen-antibody (HIV Ag-Ab) }\end{array}$ & N.A. & \multicolumn{2}{|c|}{ Non-reactive } \\
\hline Alanine transaminase (ALT) (U/L) & $10-44$ & 10 & 15 \\
\hline Aspartate transaminase (AST) (U/L) & $10-34$ & 17 & 24 \\
\hline
\end{tabular}

\section{Discussion}

TB remains a serious health problem in Singapore, especially with increasing rates of HIV and influx of migrant workers who have not been vaccinated. ${ }^{6}$ Therefore, understanding TB-associated ocular complications is important for the ophthalmologist. The occurrence of isolated ocular TB in absence of systemic TB is not unusual.' Diagnosing TB retinal vasculitis requires a high index of suspicion as its features mimics other infectious and non-infectious intraocular inflammation including sarcoidosis, Behcet's disease and isolated retinal vasculitis. ${ }^{8}$ 
According to the WHO, a definite case of TB is when Mycobacterium tuberculosis complex is identified from a clinical specimen either by culture or a newer method such as molecular line probe assay from a patient. ${ }^{1}$ However, the diagnosis of intraocular TB is difficult due to the large variations in clinical presentations, lack of uniformity in diagnostic criteria, and the low yield of organisms from the eye. One has to rely on the clinical presentations with investigations such as Mantoux test or molecular diagnostic techniques to make a diagnosis of presumed intraocular TB. ${ }^{5}$ The TB T-spot test is a reasonably fast and accurate diagnostic test. Results are obtained within 24 hours with a high sensitivity of $97.2 \%$ and a specificity of $92 \% .{ }^{9}$ It can also differentiate latent from active TB, and eliminates false positives secondary to vaccinations. ${ }^{10} \mathrm{It}$ is also found to have a stronger correlation with the level of exposure to mycobacterium tuberculosis compared to the Mantoux test. ${ }^{5}$

The 'gold standard' of TB diagnosis is culture. However, this is not feasible in patients with TB-associated uveitis without respiratory symptoms as they would be incapable of producing sputum for culture. The four to eight weeks waiting time for the culture result ${ }^{11}$ to guide the decision to initiate treatment may also be unpractical. Furthermore, culture result incubation time may prolong in multidrug resistant mycobacterium. ${ }^{12}$ The use of polymerase chain reaction (PCR) for TB in intraocular fluids is often not possible as TB particles are usually not present in TB retinal vasculitis, as it is usually immune-mediated. Additionally, aqueous or vitreous sampling may incur risks of procedural-related ocular injuries. IGRA tests such as TB T-spot test therefore hold promise as the diagnostic tool for ocular TB in the future.

The effective management of TB retinal vasculitis consists of a combination therapy of multidrug anti-TB therapy with systemic steroids. Isolated use of systemic steroids in a positive-TB patient may provoke the risk of miliary $\mathrm{TB}_{1}^{8,13}$ while isolated use of anti-TB therapy may not reduce the immune-mediated inflammation. PRP is required if there is occlusive vasculitis to prevent rubeosis from ischaemia and inflammation. ${ }^{13}$ Regular ophthalmic assessment is recommended to follow-up for resolution of fundal changes and monitoring of ocular side effects of anti-TB therapy, especially when the total dosage of ethambutol received exceeds $25 \mathrm{mg} / \mathrm{kg} .{ }^{10}$

\section{Conclusion}

TB retinal vasculitis is uncommon but demonstrating a resurgence. A high-index of suspicion with knowledge of its geographical prevalence combined with a thorough work-up to rule out other causes and multi-modal treatment is necessary for early and effective management.

\section{References}

1. World Health Organisation. Global Tuberculosis Report 2012. http://www.who.int/tb/publications/ global_report/gtbr12_main.pdf. Accessed $1^{\text {st }}$ August 2013.

2. Ministry of Health, Singapore. TB Statistics. http://www.moh.gov.sg/content/moh_web/home/ statistics/infectiousDiseasesStatistics/Tuberculosis_TB_Stats.html. Accessed $1^{\text {st }}$ August 2013.

3. Sharma A, Thapa B, Lavaju P. Ocular tuberculosis: an update. Nepal J Ophthalmol 2011;3:52-67. 
4. Sheu SJ, Shyu JS, Chen LM, Chen YY, Chirn SC, Wang JS. Ocular manifestations of tuberculosis. Ophthalmology 2001;108:1580-1585.

5. Gupta V, Gupta A, Rao N.A. Intraocular Tuberculosis- An Update. Surv Ophthalmol 2007;52:561-587.

6. Hoh HB, Kon VY, Jaais F. Tuberculous retinal vasculitis. Med J Malaysia 1998;53:288-289.

7. Sarvananthan N, Wiselka M, Bibby K et al. Intraocular tuberculosis without detectable systemic infection. Arch Ophthalmol 1998;116:1386-1388.

8. Shah SM, Sarkies NJC, Howard RS, Graham EM. Tuberculosis presenting as retinal vasculitis. J R Soc Med 1988;81:232-233.

9. Meier T, Eulenbruch HP, Wrighton-Smith P, Enders G, Regnath T. Sensitivity of a new commercial enzyme-linked immunospot assay (T SPOT-TB) for diagnosis of tuberculosis in clinical practice. Eur J Clin Microbiol Infect Dis 2005;24:529-536.

10. Pai M, Riley LW, Colford JM. Interferon-gamma assays in the immunodiagnosis of tuberculosis: a systematic review. Lancet Infect Dis 2004;4:761-776.

11. Ptyffer GE, Wittwer F. Incubation time of mycobacterial cultures: how long is long enough to issue a final negative report to clinician? J Clin Microbial 2012;50:4188-4189.

12. Giovanni BM, Alberto $M$, Daniela $C$ and Madhukar P. Diagnosis of multidrug- resistant tuberculosis and extensively drug-resistant tuberculosis: Current standards and challenges. Can J Infect Dic Med Microbiol 2008;19:169-172.

13. Kok HA, George TM, Bastion MC, Mohamad MH. Two cases of retinal vasculitis in ocular tuberculosis involving different parts of the vascular system. Med \& Health 2006;1:91-93.

14. Hoh HB, Kon VY, Jaais F. Tuberculous retinal vasculitis. Med J Malaysia 1998;53:288-289. 